\title{
ON EXTENDED SINGULAR SET OF POTENTIALS
}

\section{DARKO ŽUBRINIĆ}

Abstract. We describe a class of potentials $v=G * f$, such that if $x_{0}$ is from extended singular set of $v$, that is, $r^{-N} \int_{B_{r}\left(x_{0}\right)} v(x) d x \rightarrow+\infty$ for some sequence $r \rightarrow 0$, then necessarily $v\left(x_{0}\right)=\infty$. This class includes Bessel potentials and Riesz potentials. The result was exploited in our previous paper in order to show that singular dimension of the Bessel potential space $L^{\alpha, p}\left(\mathbf{R}^{N}\right)$ (that is, the supremum of Hausdorff's dimension of extended singular sets, taken over all functions from the space) is equal to $N-\alpha p$, provided $\alpha p \leqslant N$.

Mathematics subject classification (2000): 26A12, 31C40, 46E35.

Key words and phrases: Singular set, potentials.

\section{REFERENCES}

[1] Adams, D. R., Hedberg, L. I., Function Spaces and Potential Theory, Springer Verlag, 1996.

[2] FAlCONER, K. J., Fractal geometry, John Wiley and Sons, New York, 1990.

[3] RESHETNYAK, YU. G., On the concept of capacity in the theory of functions with generalized derivatives, Sibirskij mat. žurnal, X, 5, (1969), 1108-1138 (Russian); Siberian Math. J. 13 (1969), 818-842.

[4] ZIEMER, W. P., Weakly differentiable functions; Sobolev spaces and functions of bounded variation, Graduate texts in mathematics, Springer-Verlag, 1989.

[5] ŽUbrinić, D., Singular sets of Sobolev functions, C. R. Acad. Sci. Paris, Ser. I, 334, (2002), 539-544.

[6] ŽUBRINIĆ, D., Singular sets of Lebesgue integrable functions, Chaos, Solitons and Fractals, 21, (2004), 1281-1287. 